\title{
2D versus 3D real time ultrasound with live xPlane imaging to visualize aortic and ductal arches: comparison between methods
}

\author{
Stefania Dell'Oro $^{1}$, Maria Verderio ${ }^{1}$, Maddalena Incerti ${ }^{1}$, Salvatore Andrea Mastrolia ${ }^{\text {Corresp., }}{ }^{1}$, Sabrina \\ Cozzolino $^{1}{ }^{\text {, Patrizia Vergani }}{ }^{1}$ \\ ${ }^{1}$ Department of Maternal Fetal Medicine, Fondazione MBBM, San Gerardo Hospital, University of Milano Bicocca, Monza, Italy \\ Corresponding Author: Salvatore Andrea Mastrolia \\ Email address: mastroliasa@gmail.com
}

Background: The diagnosis of CHDs is challenging, especially for what concerns conotruncal anomalies. Indeed, although the screening techniques of fetal cardiac anomalies have greatly improved, the detection rate of conotruncal anomalies still remains low due to the fact that they are associated with a normal four-chamber view. Therefore, the study aimed to compare real-time three-dimensional echocardiography with live $x$ Plane imaging with 2D traditional imaging in visualizing ductal and aortic arches during routine echocardiography of the second trimester of gestation. Methods: This was an observational prospective study including 114 women with uncomplicated, singleton pregnancies. All sonographic studies were performed by two different operators, of them 60 by a first level operator, while 54 by a second level operator. A subanalysis was run in order to evaluate the feasibility and the time needed for the two procedures according to fetal spine position and operator's experience. Results: The measurements with 2D ultrasound were performed in all 114 echocardiographies, while live xPlane imaging was feasible in the $78 \%$ of the cases, and this was mainly due to fetal position. The time lapse needed to visualize aortic and ductal arches was significantly lower when using $2 \mathrm{D}$ ultrasound compared to live xPlane imaging $(29.56 \pm 28.5 \mathrm{sec}$ vs. $42.5 \pm 38.1 \mathrm{sec}, p=0.006$ for aortic arch; $22.14 \pm 17.8 \mathrm{sec}$ vs. $37.1 \pm 33.8 \mathrm{sec}, p=0.001$ for ductal arch), also when performing a subanalysis according to operators' experience ( $p<0.05$ for all comparisons). Feasibility of live xPlane proved to be correlated with the position of the fetal spine and the operator's experience. Discussion: To find a reproducible and standardized method to detect fetal heart defects may bring a great benefit for both patients and operators. In this scenario Live xPlane imaging is a novel method to visualize ductal and aortic arches. We found that the position of the fetal spine may affect the feasibility of the method since, when the fetal back is anterior or transverse, the visualization of the correct view of 3VT in order to set the reference line properly becomes more challenging. In addition, the fetal 
spine position influences the duration of the ultrasound examination. Regarding operator's skills and experience, in our study a first level operator was able to perform the complete 2D and xPlane examination in a lower number of cases compared to second level operators. In addition, the time required for the complete examination was higher for first level operators. This means that this technique is based on an adequate operators' expertise. 
$12 D$ versus 3D real time ultrasound with live xPlane imaging to visualize aortic and ductal

2 arches: comparison between methods

3

4 Stefania Dell'Oro ${ }^{1}$, Maria Verderio ${ }^{1}$, Maddalena Incerti ${ }^{1}$, Salvatore Andrea Mastrolia ${ }^{1}$, Sabrina

5 Cozzolino $^{1}$, Patrizia Vergani ${ }^{1}$

6

7 'Department of Maternal Fetal Medicine, Fondazione MBBM, San Gerardo Hospital, University of 8 Milano-Bicocca, Monza, Italy

9

Corresponding author: Salvatore Andrea Mastrolia, MD, Maternal Fetal Medicine Unit, Fondazione MBBM, San Gerardo Hospital, University of Milano Bicocca, Via Pergolesi 33, Monza, 20900, Monza e Brianza, Italy, Phone +390392333113, Fax +390392333131, email mastroliasa@gmail.com 


\section{Abstract}

Background: The diagnosis of CHDs is challenging, especially for what concerns conotruncal

anomalies. Indeed, although the screening techniques of fetal cardiac anomalies have greatly improved, the detection rate of conotruncal anomalies still remains low due to the fact that they are associated with a normal four-chamber view. Therefore, the study aimed to compare realtime three-dimensional echocardiography with live xPlane imaging with $2 \mathrm{D}$ traditional imaging in visualizing ductal and aortic arches during routine echocardiography of the second trimester of gestation.

Methods: This was an observational prospective study including 114 women with uncomplicated, singleton pregnancies. All sonographic studies were performed by two different operators, of them 60 by a first level operator, while 54 by a second level operator.

A subanalysis was run in order to evaluate the feasibility and the time needed for the two procedures according to fetal spine position and operator's experience.

Results: The measurements with 2D ultrasound were performed in all 114 echocardiographies, while live xPlane imaging was feasible in the $78 \%$ of the cases, and this was mainly due to fetal position. The time lapse needed to visualize aortic and ductal arches was significantly lower when using $2 \mathrm{D}$ ultrasound compared to live xPlane imaging $(29.56 \pm 28.5 \mathrm{sec}$ vs. $42.5 \pm 38.1 \mathrm{sec}$, $\mathrm{p}=0.006$ for aortic arch; $22.14 \pm 17.8 \mathrm{sec}$ vs. $37.1 \pm 33.8 \mathrm{sec}, \mathrm{p}=0.001$ for ductal arch), also when performing a subanalysis according to operators' experience ( $\mathrm{p}<0.05$ for all comparisons). Feasibility of live xPlane proved to be correlated with the position of the fetal spine and the operator's experience.

Discussion: To find a reproducible and standardized method to detect fetal heart defects may bring a great benefit for both patients and operators. In this scenario Live xPlane imaging is a 
41 novel method to visualize ductal and aortic arches. We found that the position of the fetal spine

42 may affect the feasibility of the method since, when the fetal back is anterior or transverse, the

43 visualization of the correct view of 3VT in order to set the reference line properly becomes more

44 challenging. In addition, the fetal spine position influences the duration of the ultrasound

45 examination. Regarding operator's skills and experience, in our study a first level operator was

46 able to perform the complete $2 \mathrm{D}$ and xPlane examination in a lower number of cases compared

47 to second level operators. In addition, the time required for the complete examination was higher

48 for first level operators. This means that this technique is based on an adequate operators' 49 expertise. 


\section{INTRODUCTION}

53 Congenital heart defects (CHDs) are the most common form of congenital anatomical

54 anomaly(Rocha et al. 2013).

55 They are associated with high morbidity and mortality rates, and their incidence is estimated to

56 be 4-13 per 1000 live births(Galindo et al. 2009; Sivanandam et al. 2006).

57 Antenatal screening offers a number of advantages such as establishing a strategy for peripartum

58 management and screening for co-existing abnormalities, eventually allowing intrauterine

59 intervention in some cases(Franklin et al. 2002).

60 However, the diagnosis of CHDs is challenging, especially for what concerns conotruncal

61 anomalies. Indeed, although the screening techniques of fetal cardiac anomalies have greatly

62 improved, the detection rate of conotruncal anomalies still remains low due to the fact that they

63 are associated with a normal four-chamber view(Paladini et al. 1996; Sivanandam et al. 2006;

64 Tometzki et al. 1999). At the beginning, the study of the fetal heart was based only on four-

65 chamber view, while today, international guidelines(International Society of Ultrasound in et al.

66 2013) consider left and right ventricular outflow tracts (LVOT and RVOT, respectively) as an

67 important part of fetal cardiac screening examination, while three-vessels (3V), and three-vessels

68 and trachea (3VT) are not mandatory due to the technically challenging acquisition and

69 interpretation(International Society of Ultrasound in \& Gynecology 2006; International Society

70 of Ultrasound in et al. 2013), although strongly recommended in order to increase and improve

71 operators' confidence with these scans.

72 The importance of improving the detection rate of CHDs, with special attention to conotruncal

73 anomalies assessing the $3 \mathrm{VT}$, as well as the aortic and ductal arches, in addition to the four-

74 chamber view, LVOT and RVOT, was suggested by several authors(Allan 2004; Espinoza et al. 
75

76

77

78

79

80

81

82

83

84

85

86

87

88

89

90

91

92

93

94

95

96

2007b).

However, performing all these sonographic scans needs systematic training and may depend on the operators' skills and experience.

According to the above mentioned literature, there is a need to find a method that could improve the detection rate for CHDs, and especially of conotruncal anomalies, taking into consideration the possibility of a modification in the spatial relationships among cardiac chambers and great vessels throughout gestation(Espinoza et al. 2007a). This geometric three-dimensional change can be even more prominent in case of cardiac anomalies(Yuan et al. 2011), although the study of cardiac anomalies is beyond the scope of the present study.

Live xPlane imaging, a new kind of real-time three-dimensional echocardiography, using a matrix-array probe, enabling the visualization of the pulsating fetal heart in real time, akin to real-time gray-scale scanning(Acar et al. 2005; Sugeng et al. 2003; Xiong et al. 2012a; Xiong et al. 2012b; Xiong et al. 2013a; Xiong et al. 2013b; Xiong et al. 2009; Yuan et al. 2011), might be useful to the purpose of improving the detection rate of CHDs according to geometric threedimensional changes of the fetal heart. In fact, this modality allows the simultaneous visualization of real-time high-resolution view of two planes, oriented in different directions, giving a satisfactory spatial resolution(Taddei et al. 2007).

92 Therefore, the objective of the present study was to evaluate to feasibility of real-time three-

93 dimensional echocardiography with live xPlane imaging compared to $2 \mathrm{D}$ traditional imaging in

94 visualizing ductal and aortic arches, analyzing the potential impact of factors influencing the 95 feasibility of the technnique, such as position of the fetal spine and operator's experience and skills. 


\section{MATERIAL AND METHODS}

$98 \quad$ Setting and eligibility criteria

99 This was a prospective observational study including all women with singleton pregnancies from $10018+1$ to $21+6$ weeks of gestation, attending routine echocardiography of the second trimester, 101 between March 2015 and December 2016 at our Department of Maternal Fetal Medicine 102 (Fondazione MBBM, San Gerardo Hospital, University of Milano Bicocca, Monza, Italy). 103 Women with indications for fetal echocardiography (chromosomal abnormalities, abnormal first 104 trimester screening, fetal malformations, hereditary maternal diseases associated with cardiac 105 defects, maternal infections, maternal disorders such as pregestational diabetes, phenylketonuria, 106 autoimmune diseases, use of drugs with potential teratogenicity), were excluded from the study.

107 Medical and obstetrical history was investigated before enrollment in order to find any exclusion 108 criteria. One hundred and fourteen women were enrolled in the study after meeting the inclusion 109 criteria and accepting to participate. Informed consent was obtained in all cases.

\section{Data collection}

111 All ultrasound examinations were performed by using iU22 ultrasound scanner (Philips Medical

112 System), with two different probes: for the 2D ultrasound, the C5-1 probe was used, while the 113 3D scan was performed with the matrix X6-1transducer.

114 The ultrasound scans were executed alternatively by two sonographers: a first level operator and 115 a second level operator. Operators were defined according to the Report of WHO Study Group 116 on training in diagnostic ultrasound. Therefore, the first level operator was defined as able to 117 a) perform common examinations safely and accurately; b) recognize and differentiate normal 118 anatomy and pathology; c) diagnose common abnormalities within certain organ systems; d) 119 recognize when referral for a second opinion is indicated. The second level operator was defined 
120 as a specialist in Fetal Medicine able to a) accept and manage referrals from Level 1 121 practitioners; b) recognize and correctly diagnose almost all pathology within the relevant organ 122 systems; c) perform basic, non-complex ultrasound-guided invasive procedures; d) teach 123 ultrasound to trainees and to Level 1 practitioners; e) conduct some research in ultrasound.

124 The first step of sonographic evaluation was the description of the position of the fetal spine.

125 Then, all women underwent 2D ultrasound and, starting from the four-chamber view, the time to 126 visualize ductal and aortic arches in longitudinal section was measured.

127 The criteria for a successful imaging of the aortic arch view were that the whole course of the 128 ascending aorta, transverse aortic arch, aortic isthmus and upper part of descending aorta were 129 visualized in continuity (Figure 1). The criteria for the successful imaging of the ductal arch were 130 the visualization of continuity of the right ventricle, pulmonary valve, pulmonary artery, ductus 131 arteriosus and upper part of the descending aorta, together with the short axis of ascending aorta 132 (Figure 2).

133 The detailed method of visualization of the aortic arch and the ductal arch view with live xPlane 134 imaging was defined as follows. A successful demonstration of the arches using live xPlane 135 imaging required the correct orientation of the great vessels in the $3 \mathrm{VT}$ view. For this reason, the 136 first step was to obtain the four-chamber view, then slightly tilting the transducer towards the 137 fetal head in order to visualize the 3VT view. After that, the two planes were displayed in real 138 time by the activation of live xPlane imaging function. The original two-dimensional image was 139 visualized on the left side of the screen (primary plane). The transducer was then moved in a way 140 that either the pulmonary artery or the aorta was lying parallel to the direction of the ultrasound

141 beam. A secondary plane across the reference line was displayed in the right window by moving 142 the reference line on the primary plane. In our study, the 3VT plane was displayed on the left 
143 side. The reference line was adjusted in the primary plane to be placed along the center of the

144 pulmonary trunk and descending aorta in the $3 \mathrm{VT}$ view, allowing the ductal arch view to be

145 displayed in the right window. By the slight adjustment of the position of the transducer, moving

146 the reference line through the center of the transverse aortic arch and descending aorta in the

$1473 \mathrm{VT}$ views, the aortic-arch was visualized within the right window.

148 In our study, depending on fetal position, we displayed the aortic arch view at first and then the 149 ductal arch view.

150 The exam was defined not doable when the two arches were not visualized within five minutes.

151 The study has been performed in accordance with the ethical standards laid down in the 1964

152 Declaration of Helsinki and its later amendments (Helsinki Declaration 1975, revision 2013) and

153 approved by the Institutional Review Board Committee of San Gerardo Hospital (Monza, 154 Italy)(IRB No. 3022015).

155 Statistical analysis was performed with IBM SPSS Statistic version 21 (IBM Corp. Released 156 2012. IBM SPSS Statistics for Windows, Version 21.0. Armonk, NY: IBM Corp). Data on 157 continuous variables with normal distribution were presented as mean $\pm \mathrm{SD}$, and compared 158 between study groups using Student t-test. Continuous variables not normally distributed and 159 ordinal variables were presented as median with inter-quartile range (IQ range), and statistical 160 analysis was performed using Mann-Whitney test. Categorical data were shown in numbers and 161 percentages, while their differences were assessed by Chi-Square. Fisher Exact test was used 162 when appropriate. A p-value $<0.05$ was considered as statistically significant. 


\section{RESULTS}

164 A total of 114 pregnant women of different ethnic groups were enrolled in this study. The mean 165 maternal age was 33.07 \pm 5.23 (range 19-45) years with a mean body mass index of $24.82 \pm 4.17$ 166 (range 17.74- 39.3). The mean gestational age at sonographic examination was $20.3 \pm 0.5$ weeks 167 (range 18.6-21.5).

168 In 6 cases $(5.3 \%)$ the fetus was in transverse lie, while in 108 exams $(94.7 \%)$ fetuses were in 169 longitudinal lie, of them 62 cases in vertex presentation $(57.4 \%)$ and 46 in breech presentation $170(42.6 \%)$.

171 The visualization rate of the aortic arch and ductal arch with $2 \mathrm{D}$ ultrasound was performed in all 172114 cases $(100 \%)$, while the examination was completed in 89 cases $(78.1 \%)$ using live xPlane 173 imaging, of them 92 with aortic arch examination (80.7\%) and 93 ending with ductal arch 174 examination $(81.5 \%)$ (Table 1$)$.

175 Table 2 shows a comparison between the time averages required with the two different methods. 176 The timing with 2D ultrasound was significantly lower compared to live xPlane imaging starting 177 from the four-chamber view until visualization of either aortic $(29.56 \pm 28.5 \mathrm{sec}$ vs. $42.5 \pm 38.1$ 178 sec, $\mathrm{p}=0.006)$ as well as ductal arch $(22.14 \pm 17.8 \mathrm{sec}$ vs. $37.1 \pm 33.8 \mathrm{sec}, \mathrm{p}=0.001)$.

179 Compared to 2D ultrasound, live xPlane was more doable when the fetal back was in the right $180(100 \%$ vs. $100 \%)$, posterior (100\% vs. $100 \%)$ or in left $(100 \%$ vs. $93.8 \%)$ longitudinal position, 181 while the feasibility was very low when the fetal back was anterior (100\% vs. $24 \%)$ or transverse 182 (100\% vs. 33.3\%) (Table 3).

183 In addition to feasibility of both techniques, a comparison of the time averages to perform 2D 184 ultrasound vs. xPlane was done according to fetal spine position. Compared to live xPlane, 2D 185 ultrasound was faster when the fetal back was in anterior $(55.65 \pm 34.14 \mathrm{sec}$ vs. $139.5 \pm 62.84 \mathrm{sec}$, 
$186 \mathrm{p}=0.002)$, or in right longitudinal position $(42.78 \pm 28.65 \mathrm{sec}$ vs. $73.29 \pm 50.64 \mathrm{sec}, \mathrm{p}$ value $=0.023)$,

187 while it was slower when the back was in inferior position $(80.33 \pm 45.85 \mathrm{sec}$ vs. $46.0 \pm 12.72 \mathrm{sec}$, $188 \mathrm{p}=0.042)$. When the spine was posterior $(44.72 \pm 25.33 \mathrm{sec}$ vs. $48.87 \pm 51.58 \mathrm{sec}$, $\mathrm{p}$ value $=0.256)$ or 189 in left position $(55.75 \pm 41.99 \mathrm{sec}$ vs. $64.0 \pm 38.1 \mathrm{sec}, \mathrm{p}$ value $=0.377)$, times to achieve the scans 190 were similar (Table 4).

191 Echocardiographies were performed by two different operators: $60(52.6 \%)$ of them were 192 performed by a first level operator, while 54 (47.4\%) were executed by a second level operator.

193 The first level operator, had a successful visualization rate of the aortic and ductal arch with 2D 194 ultrasound in all 60 cases (100\%), while the complete live xPlane imaging was performed only in 19538 cases (63.3\%), of them 40 with aortic arch examination (66.6\%) and 41 ending with ductal 196 arch examination (68.3\%). Regarding the second level operator, the visualization of the aortic 197 and ductal arch with 2D ultrasound was performed in all 54 cases, while it was possible to 198 perform aortic arch examination in 52 cases $(96.29 \%)$ and ductal arch examination in another 52 199 women (96.29\%), having the complete live xPlane imaging in 51 cases $(94.4 \%)$ (Table 5).

The comparison between the time averages required with the two different methods, according to 201 operator's level is shown in Table 6. A higher amount of time to perform the examination with 202 live xPlane compared to 2D ultrasound was required for both first level as well as second level operator. For the first level operator, the time with 2D ultrasound was significantly lower compared to live xPlane imaging starting from the four-chamber view until visualization of either aortic $(30.25 \pm 29.25 \mathrm{sec}$ vs. $51.62 \pm 36.89 \mathrm{sec}, \mathrm{p}=0.002)$ as well as ductal arch $(25.51 \pm 20.62$ sec vs. $39.09 \pm 34.23 \mathrm{sec}, \mathrm{p}=0.03$ ). Also for second level operator, the time needed to visualize the aortic arch using 2D ultrasound was significantly lower $(28.79 \pm 28.17$ sec vs. $35.5 \pm 37.9$ sec, $\mathrm{p}=0.02)$ as was for ductal arch $(18.38 \pm 13.21 \mathrm{sec}$ vs. $35.5 \pm 33.8 \mathrm{sec}, \mathrm{p}=0.001)$, compared to live 
209 xPlane.

210 Figure 3 shows the distribution of the times with 2D vs. 3D live xPlane, related with the spine

211 position and divided according to operator's level. The median for the $2 \mathrm{D}$ method was always

212 lower or similar to 3D live xPlane, for both the first and second level operator. This was true

213 except when the fetal back was posterior since, in this case, the second level operator performed

214 live xPlane in a lower time compared to 2D ultrasound. 


\section{DISCUSSION}

216 Principal findings of the study: 1) Live xPlane imaging allows a better identification of the

217 aortic and ductal arches without the risk to confound them, while this is not true for the $2 \mathrm{D}$

218 method where the longitudinal visualization of the arches may mislead the operator due to their

219 similar characteristics; 2) This technique can be useful in fetal echocardiography during the

220 second trimester executed by an experienced operator; and 3) The time required to complete live

221 xPlane examination compared to 2D ultrasound examination of the fetal heart is dependent on

222 both fetal position and operator experience.

223 The need for additional techniques in the screening for CHDs: Although screening of CHDs

224 has developed greatly in the last years, according to new guidelines, the detection rate of fetal

225 conotruncal anomalies is still low(Galindo et al. 2009; Paladini et al. 1996; Sivanandam et al.

226 2006; Tometzki et al. 1999), especially among non-experienced operator(Tegnander \& Eik-Nes

227 2006). This is mainly because these anomalies usually are associated with a normal four-

228 chamber view. Detection rate can only be increased by including additional views of the outflow

229 tracts, as recommended both for SIEOG and ISUOG guidelines(International Society of

230 Ultrasound in et al. 2013). This would further include left and right outflow tract view and 3VT.

231 However, realizing these four views is not easy and needs systematic training and depends on the

232 operators' experience(Tegnander \& Eik-Nes 2006). Therefore, to find a simple, reproducible,

233 and standardized method to detect fetal heart defects may bring a great benefit for both patients

234 and operators, especially non-experienced ones.

235 3VT views can be obtained as easily as the four chamber-view, which may be useful as a

236 complementary view in routine screening for CHD(Vinals et al. 2003; Yagel et al. 2002).

237 However, according to the available literature, which proposes the use of $3 \mathrm{VT}$ in routine 
238 screening, we decided to study the aortic and ductal arches with live xPlane, since easily

239 obtainable from the 3VT, despite the study of fetal heart does not include the study of aortic and

240 ductal arches. In fact, aortic and ductal arch are a useful tool to screen for conotruncal

241 anomalies(Espinoza et al. 2007b).

242 Some researchers proposed to use spatiotemporal image correlation (STIC), a form of

243 reconstructed 3D echocardiography, to improve the detection rate of fetal conotruncal

244 anomalies(DeVore et al. 2003; Espinoza et al. 2007b; Goncalves et al. 2006; Yagel et al. 2002).

245 However, this method is influenced by fetal breathing and movements and is subjected to

246 movement artifacts (Goncalves et al. 2003).

247 Conversely, live xPlane, based on real-time 3D processing, allows simultaneous display of two

248 planes in real time and, for this reason, is not associated to artifacts.

249 Live xPlane imaging and its utility according to fetal cardiac geometry: Live xPlane imaging

250 is described as a novel and relatively simple method, with a good sensibility and specificity,

251 simple to teach and learn(Xiong et al. 2012a).The application of a matrix probe in scanning fetal

252 heart had been reported by several researchers(Goncalves et al. 2003; Goncalves et al. 2006), but

253 there are only few studies providing a detailed description of its methodology in the visualization

254 of the recommended sections of the fetal heart(Xiong et al. 2012a; Xiong et al. 2012b; Xiong et

255 al. 2013a; Xiong et al. 2013b; Xiong et al. 2009; Yuan et al. 2011).

256 In the available literature, live xPlane has been employed to visualize some specific cardiac

257 structures, in order to improve the detection rate of CDHs. For example, Xiong et al(Xiong et al.

258 2013a; Xiong et al. 2009)evaluated the feasibility of this method for the visualization of

259 interventricular septum, while Yuan et al(Yuan et al. 2011)used live xPlane to examine

260 simultaneously the four-chamber, the LVOT and the angle between these structures. 
261 Furthermore, Xiong et al(Xiong et al. 2012b) reported the methodology of acquiring and

262 examining the screening planes, with the advantages and disadvantages of this method.

263 Yuan et al(Yuan et al. 2011), use xPlane to study the rotation angle from four-chamber view to

264 LVOT and investigate factors affecting the angles: they describe how abnormal rotation angles

265 and angle span may be associated with congenital heart disease with conotruncal disorders.

266 Instead, Espinoza et al. describe how the fetal cardiac geometry occurs during the gestation,

267 particularly before 26 weeks of gestation. These observations indicate that the spatial

268 relationships between cardiac chambers and great vessels are not constant throughout

269 gestation(Espinoza et al. 2007a).

270 What is the time needed for the evaluation of the aortic and ductal arches? Live xPlane is

271 strongly related with the spine position and the fetal movements. In comparison with 2D

272 ultrasound, it requires a longer time average because, in order to get the aortic and ductal arches,

273 the reference line should be placed in the center of the pulmonary trunk and in the aorta. There is

274 no literature analyzing the time average needed to obtain these scans, as we did in the present

275 study.

276 What is the effect of fetal spine position in the evaluation of the aortic and ductal arches?

277 Feasibility of live xPlane proved to be strongly correlated with position of the fetal spine.

278 Literature suggests live xPlane to be feasible in $100 \%$ of cases, but the available studies were

279 performed when the back was posterior only(Xiong et al. 2013b). No studies correlated the

280 feasibility of the technique with the fetal spine position. Indeed, when the back is anterior or

281 transverse, the feasibility is very low, because of the difficulty to visualize the correct view of

$2823 \mathrm{VT}$ in a way to apply the method putting correctly the reference line, as we observed in our

283 study. Of interest, as described within our study, the fetal spine position influences the duration 
284 of the ultrasound examination.

285 Strengths and limitations of the study: The main advantage of live xPlane imaging is that it is 286 relatively simple and that there are no movement artifacts because live $\mathrm{x}$ Plane imaging is 287 performed in real time. Major limitation of this study is that the reference line only comes from 288 the midline of the transducer and cannot be manipulated from all directions. In fact, the reference 289 line could only be placed in parallel position concerning the ultrasound beam. This is the reason 290 why, when the fetal back is anterior or transverse, it is really difficult to obtain the visualization 291 of the arches. Thus, if the fetus is in an unfavorable position, the acquisition time will be much 292 longer. 


\section{CONCLUSIONS}

294 Live xPlane it is a relatively simple method and may potentially be a useful tool in the ultrasound 295 evaluation for the diagnosis of fetal conotruncal anomalies allowing a visualization of the aortic 296 and ductal arches and improving the detection rate of CHDs. This is especially true for 297 contruncal anomalies, taking into consideration the possibility of a modification in the spatial 298 relationships among cardiac chambers and great vessels throughout gestation(Espinoza et al. 299 2007a) and the fact that this geometric three-dimensional change can be even more prominent in 300 case of cardiac anomalies(Yuan et al. 2011), although the study of cardiac anomalies is beyond 301 the scope of the present study. Live xPlane is dependent on the position of the fetal spine and 302 needs an adequately trained operator in order to be performed. Increasing the operators' expertise 303 is crucial in order to improve the detection of cardiac anomalies using this innovative technique. 
Linee Guida SIEOG. Societa' Italiana di Ecografia Ostetrica e Ginecologica. Editeam Edizioni, 2006.

Linee Guida SIEOG. Societa' Italiana di Ecografia Ostetrica e Ginecologica. Editeam Edizioni, 2013.

Linee Guida SIEOG. Societa' Italiana di Ecografia Ostetrica e Ginecologica. Editeam Edizioni, 2015.

Training in Diagnostic Ultrasound: Essentials, Principles and Standards: Report of WHO Study Group 1998. WHO technical report series: 875

Acar P, Dulac Y, Taktak A, and Abadir S. 2005. Real-time three-dimensional fetal echocardiography using matrix probe. Prenat Diagn 25:370-375. 10.1002/pd.1159

Allan L. 2004. Technique of fetal echocardiography. Pediatr Cardiol 25:223-233. 10.1007/s00246-0030588-y

DeVore GR, Falkensammer P, Sklansky MS, and Platt LD. 2003. Spatio-temporal image correlation (STIC): new technology for evaluation of the fetal heart. Ultrasound Obstet Gynecol 22:380-387. 10.1002/uog.217

Espinoza J, Gotsch F, Kusanovic JP, Goncalves LF, Lee W, Hassan S, Mittal P, Schoen ML, and Romero R. 2007a. Changes in fetal cardiac geometry with gestation: implications for 3- and 4dimensional fetal echocardiography. J Ultrasound Med 26:437-443; quiz 444.

Espinoza J, Romero R, Kusanovic JP, Gotsch F, Erez O, Lee W, Goncalves LF, Schoen ML, and Hassan SS. 2007b. The role of the sagittal view of the ductal arch in identification of fetuses with conotruncal anomalies using 4-dimensional ultrasonography. $J$ Ultrasound Med 26:1181-1188; quiz 1189-1190.

Franklin O, Burch M, Manning N, Sleeman K, Gould S, and Archer N. 2002. Prenatal diagnosis of coarctation of the aorta improves survival and reduces morbidity. Heart 87:67-69.

Galindo A, Mendoza A, Arbues J, Graneras A, Escribano D, and Nieto O. 2009. Conotruncal anomalies in fetal life: accuracy of diagnosis, associated defects and outcome. Eur J Obstet Gynecol Reprod Biol 146:55-60. 10.1016/j.ejogrb.2009.04.032

Goncalves LF, Lee W, Chaiworapongsa T, Espinoza J, Schoen ML, Falkensammer P, Treadwell M, and Romero R. 2003. Four-dimensional ultrasonography of the fetal heart with spatiotemporal image correlation. Am J Obstet Gynecol 189:1792-1802.

Goncalves LF, Lee W, Espinoza J, and Romero R. 2006. Examination of the fetal heart by fourdimensional (4D) ultrasound with spatio-temporal image correlation (STIC). Ultrasound Obstet Gynecol 27:336-348. 10.1002/uog.2724

International Society of Ultrasound in O, and Gynecology. 2006. Cardiac screening examination of the fetus: guidelines for performing the 'basic' and 'extended basic' cardiac scan. Ultrasound Obstet Gynecol 27:107-113. 10.1002/uog.2677

International Society of Ultrasound in O, Gynecology, Carvalho JS, Allan LD, Chaoui R, Copel JA, DeVore GR, Hecher K, Lee W, Munoz H, Paladini D, Tutschek B, and Yagel S. 2013. ISUOG Practice Guidelines (updated): sonographic screening examination of the fetal heart. Ultrasound Obstet Gynecol 41:348-359. 10.1002/uog.12403

Paladini D, Rustico M, Todros T, Palmieri S, Gaglioti P, Benettoni A, Russo MG, Chiappa E, and D'Ottavio G. 1996. Conotruncal anomalies in prenatal life. Ultrasound Obstet Gynecol 8:241246. 10.1046/j.1469-0705.1996.08040241.x

Rocha LA, Araujo Junior E, Nardozza LM, and Moron AF. 2013. Screening of fetal congenital heart disease: the challenge continues. Rev Bras Cir Cardiovasc 28:V-VII. 10.5935/16789741.20130048

Sivanandam S, Glickstein JS, Printz BF, Allan LD, Altmann K, Solowiejczyk DE, Simpson L, PerezDelboy A, and Kleinman CS. 2006. Prenatal diagnosis of conotruncal malformations: diagnostic accuracy, outcome, chromosomal abnormalities, and extracardiac anomalies. Am J Perinatol 23:241-245. 10.1055/s-2006-939535 
352
Sugeng L, Weinert L, Thiele K, and Lang RM. 2003. Real-time three-dimensional echocardiography using a novel matrix array transducer. Echocardiography 20:623-635.

Taddei F, Franceschetti L, Farina G, Prefumo F, Signorelli M, Fratelli N, and Groli C. 2007. Matrix Array Trasducers in Fetal Heart Imaging. Donald School Journal of Ultrasound in Ostetrics and Gynecology 1:45-48.

Tegnander E, and Eik-Nes SH. 2006. The examiner's ultrasound experience has a significant impact on the detection rate of congenital heart defects at the second-trimester fetal examination. Ultrasound Obstet Gynecol 28:8-14. 10.1002/uog.2804

Tometzki AJ, Suda K, Kohl T, Kovalchin JP, and Silverman NH. 1999. Accuracy of prenatal echocardiographic diagnosis and prognosis of fetuses with conotruncal anomalies. J Am Coll Cardiol 33:1696-1701.

Vinals F, Heredia F, and Giuliano A. 2003. The role of the three vessels and trachea view (3VT) in the diagnosis of congenital heart defects. Ultrasound Obstet Gynecol 22:358-367. 10.1002/uog.882

Xiong Y, Chen M, Chan LW, Ting YH, Fung TY, Leung TY, and Lau TK. 2012a. A novel way of visualizing the ductal and aortic arches by real-time three-dimensional ultrasound with live xPlane imaging. Ultrasound Obstet Gynecol 39:316-321. 10.1002/uog.9081

Xiong Y, Chen M, Chan LW, Ting YH, Fung TY, Leung TY, and Lau TK. 2012b. Scan the fetal heart by real-time three-dimensional echocardiography with live xPlane imaging. J Matern Fetal Neonatal Med 25:324-328. 10.3109/14767058.2011.575904

Xiong Y, Liu T, Gan HJ, Wu Y, Xu JF, Ting YH, Leung TY, and Lau TK. 2013a. Can live xPlane imaging of the in-plane view of interventricular septum be used to detect fetal conotruncal anomalies? J Matern Fetal Neonatal Med 26:373-377. 10.3109/14767058.2012.733762

Xiong Y, Liu T, Gan HJ, Wu Y, Xu JF, Ting YH, Leung TY, and Lau TK. 2013b. Detection of the fetal conotruncal anomalies using real-time three-dimensional echocardiography with live xPlane imaging of the fetal ductal arch view. Prenat Diagn 33:462-466. 10.1002/pd.4088

Xiong Y, Wah YM, Chen M, Leung TY, and Lau TK. 2009. Real-time three-dimensional echocardiography using a matrix probe with live xPlane imaging of the interventricular septum. Ultrasound Obstet Gynecol 34:534-537. 10.1002/uog.7337

Yagel S, Arbel R, Anteby EY, Raveh D, and Achiron R. 2002. The three vessels and trachea view (3VT) in fetal cardiac scanning. Ultrasound Obstet Gynecol 20:340-345. 10.1046/j.14690705.2002.00801.x

Yuan Y, Leung KY, Ouyang YS, Yang F, Tang MH, Chau AK, and Dai Q. 2011. Simultaneous real-time imaging of four-chamber and left ventricular outflow tract views using xPlane imaging capability of a matrix array probe. Ultrasound Obstet Gynecol 37:302-309. 10.1002/uog.8883 
388 Figure legends

389

390 Figure 1. Aortic arch view, using live xPlane imaging. On the left, the 3VT view, on the right, the 391 aortic arch view, obtained by this new method.

392

393 Figure 2. Ductal arch view, using live xPlane imaging. On the left, 3VT view, on the right, the

394 ductal arch view, obtained by this new method

395

396 Figure 3. Distribution of the times with 2D vs. 3D live xPlane, related with the spine position and

397 divided according to operator's level

398 


\section{Figure 1}

Aortic arch view, using live xPlane imaging. On the left, the 3VT view, on the right, the aortic arch view, obtained by this new method.

*Note: Auto Gamma Correction was used for the image. This only affects the reviewing manuscript. See original source image if needed for review.

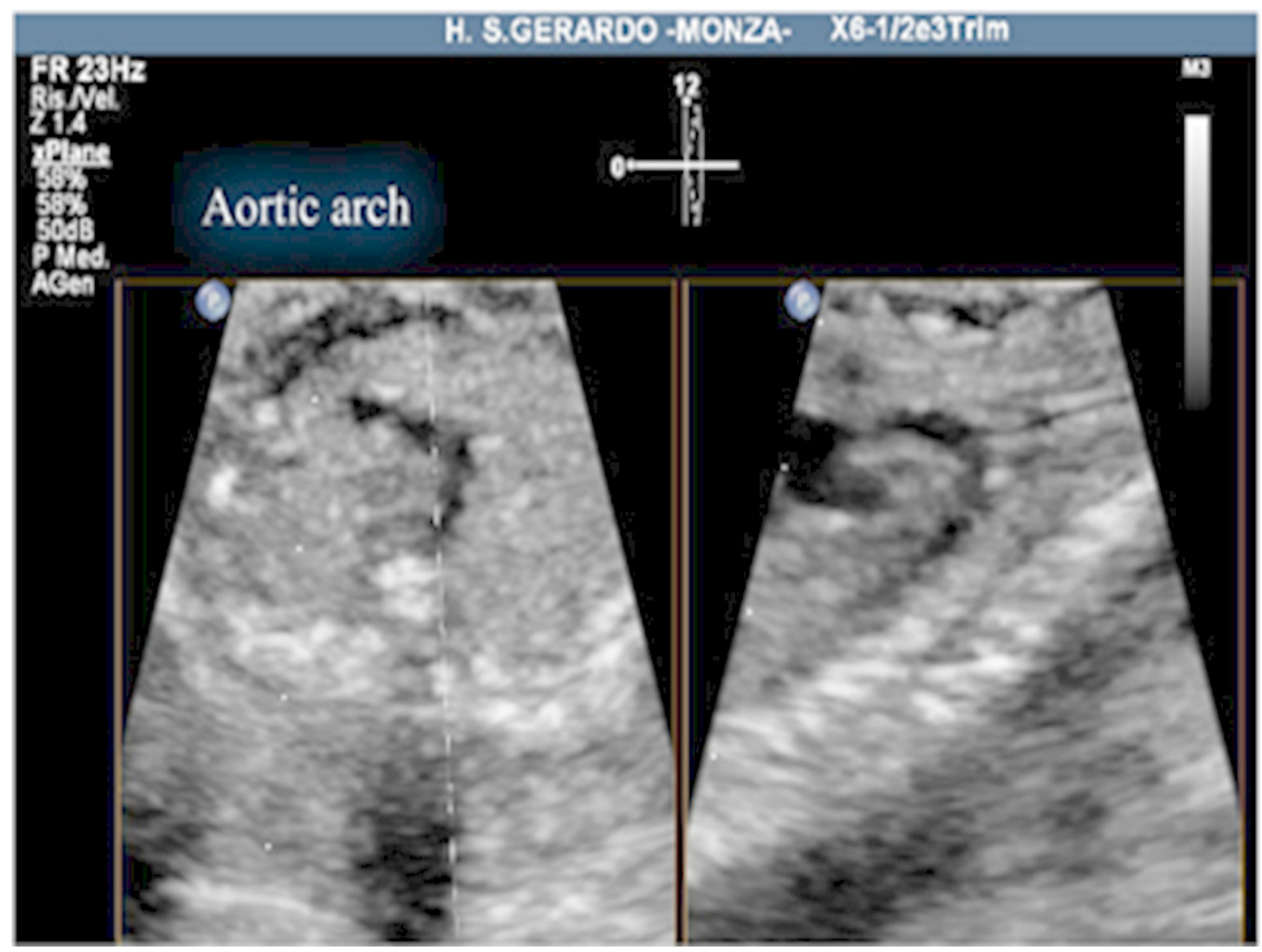




\section{Figure 2}

Ductal arch view, using live xPlane imaging. On the left, 3VT view, on the right, the ductal arch view, obtained by this new method

*Note: Auto Gamma Correction was used for the image. This only affects the reviewing manuscript. See original source image if needed for review.

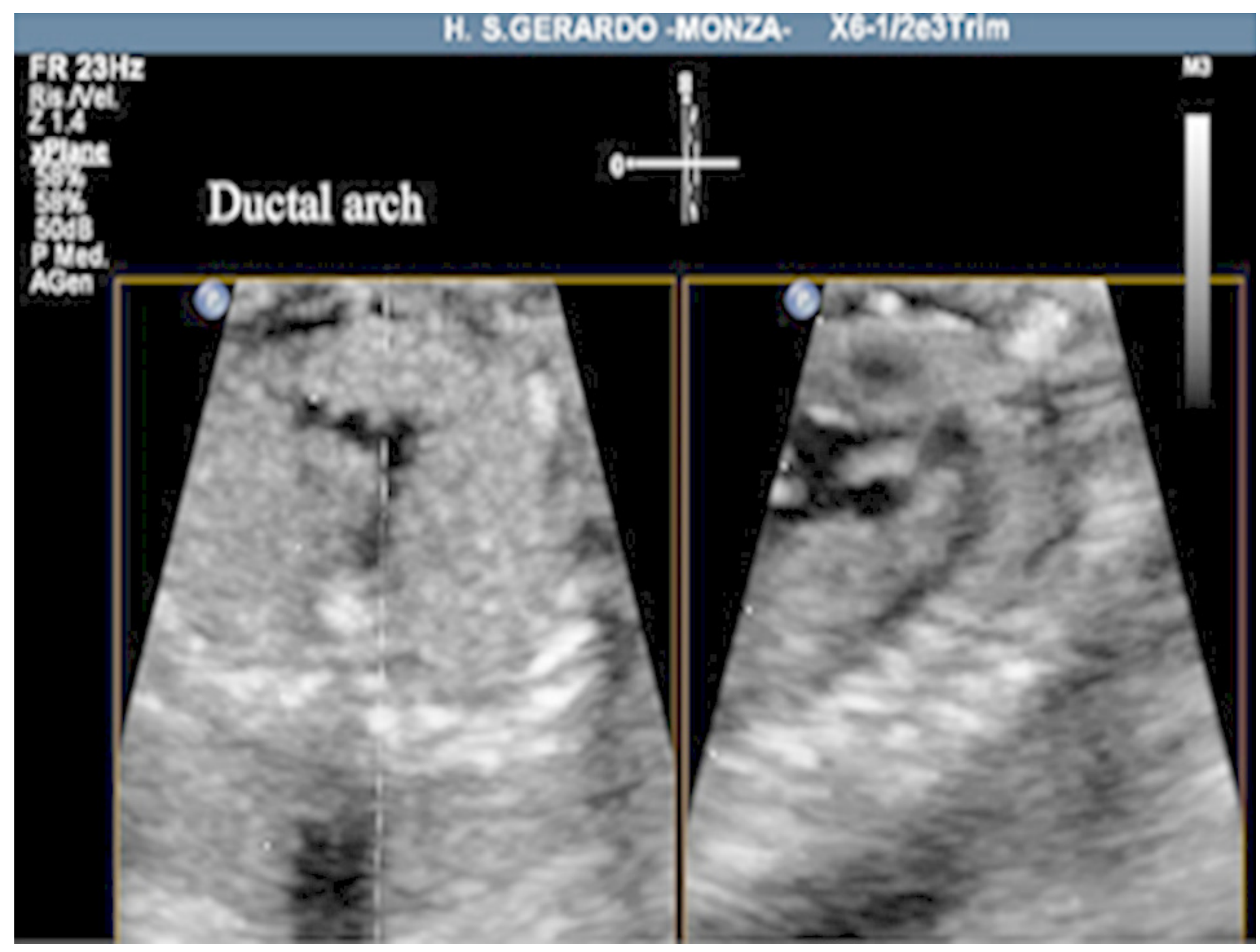


Figure 3

Distribution of the times with 2D vs. 3D live xPlane, related with the spine position and divided according to operator's level

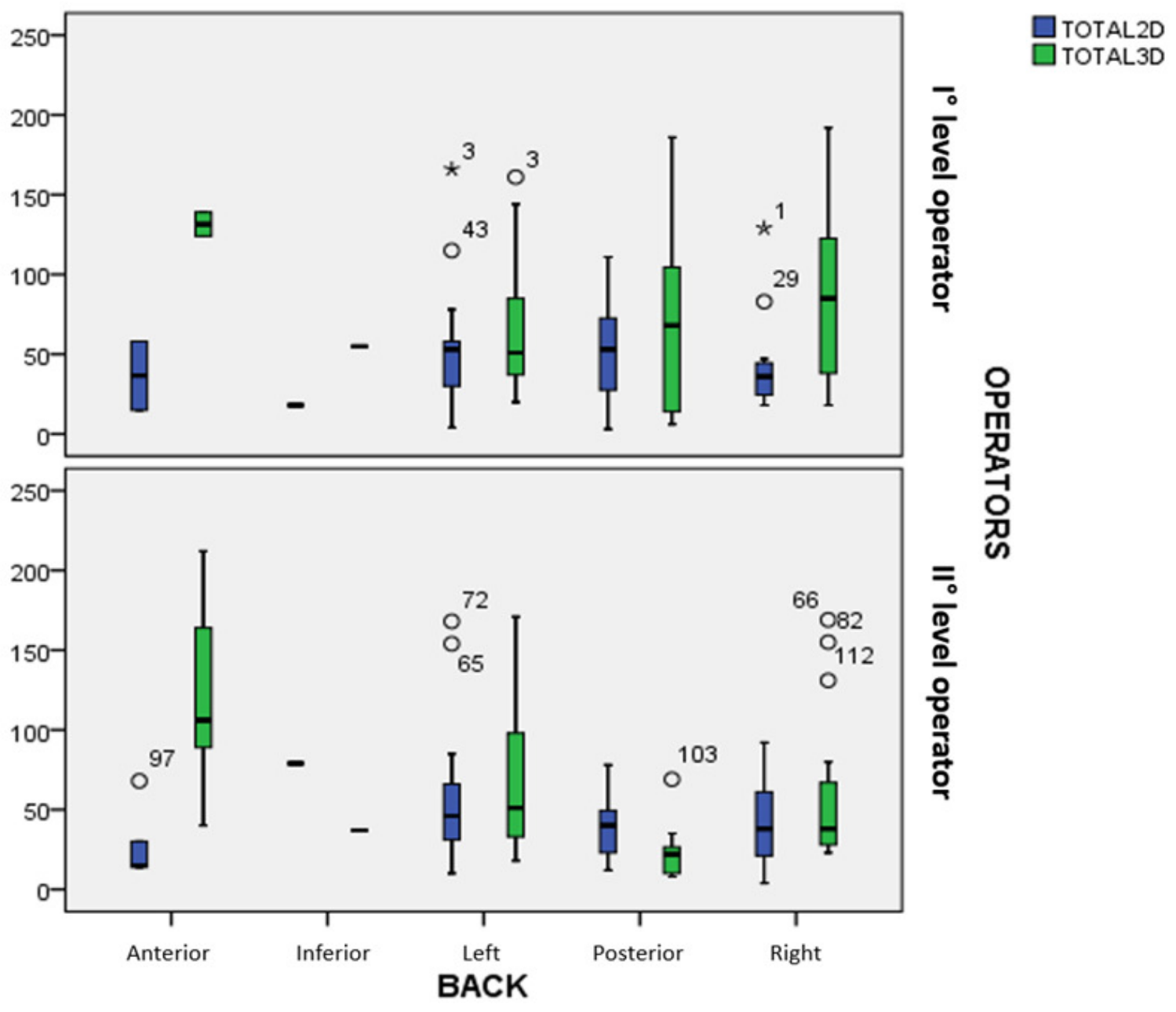




\section{Table $\mathbf{1}$ (on next page)}

Feasibility with 2D ultrasound and live xPlane

Data are presented as number (percentage). 
Table 1. Feasibility with 2D ultrasound and live xPlane.

\begin{tabular}{|c|c|c|c|}
\hline & $\begin{array}{c}\text { Feasibility 2D } \\
(\mathbf{n}=114)\end{array}$ & Feasibility live xPlane & P value \\
$(\mathbf{n}=114)$ & & $<0.001$ \\
\hline $\begin{array}{l}4 \text { Chamber- } \\
\text { Aortic Arch }\end{array}$ & $114(100 \%)$ & $92(80.7 \%)$ & Data \\
\hline $\begin{array}{l}4 \text { Chamber- } \\
\text { Ductal Arch }\end{array}$ & $114(100 \%)$ & $93(81.5 \%)$ & $<0.001$ \\
\hline
\end{tabular}

presented as number (percentage) 


\section{Table 2 (on next page)}

Average of times with 2D ultrasound and live xPlane

Data is presented as mean $\pm \mathrm{SD}$. 
Table 2. Average of times with 2D ultrasound and live xPlane.

\begin{tabular}{|c|c|c|c|}
\hline & 2D ( $\mathbf{n = 1 1 4 )}$ & Live xPlane $(\mathbf{n = 8 9})$ & P value \\
\hline $\begin{array}{c}\text { 4 Chamber- } \\
\text { Aortic Arch (sec) }\end{array}$ & $29.56 \pm 28.5$ & $42.5 \pm 38.1$ & 0.006 \\
\hline $\begin{array}{c}4 \text { Chamber- } \\
\text { Ductal Arch (sec) }\end{array}$ & $22.14 \pm 17.8$ & $37.1 \pm 33.8$ & 0.001 \\
\hline
\end{tabular}

Data is presented as mean $\pm S D$ 


\section{Table 3(on next page)}

Feasibility of 2D ultrasound vs. live xPlane according to fetal spine position

Data is presented as number (percentage). 
Table 3. Feasibility of 2D ultrasound vs. live xPlane according to fetal spine position.

\begin{tabular}{|l|c|c|c|}
\hline Fetal Position & 2D (n=114) & Live xPlane (n=114) & P value \\
\hline Anterior & $26 / 26(100)$ & $6 / 25(24)$ & $<0.001$ \\
\hline Posterior & $22 / 22(100)$ & $24 / 24(100)$ & 1 \\
\hline Right & $28 / 28(100)$ & $27 / 27(100)$ & 1 \\
\hline Left & $32 / 32(100)$ & $30 / 32(93.8)$ & 0.856 \\
\hline Inferior & $6 / 6(100)$ & $2 / 6(33.3)$ & $<0.001$ \\
\hline & $0 / 0(0)$ & $0 / 0(0)$ & $n / a$ \\
\hline Superior & $114 / 114(100)$ & $89 / 114(78.07)$ & $<0.001$ \\
\hline \multicolumn{2}{|r|}{} \\
TOTAL & \multicolumn{2}{|c|}{} \\
\hline
\end{tabular}

Data is presented as number (percentage) 


\section{Table 4 (on next page)}

Average times need for 2D ultrasound vs. live xPlane according to fetal spine position

Data is presented as number (percentage). 


\begin{tabular}{|l|c|c|c|}
\hline Fetal Position/Time (sec) & 2D (n=114) & Live xPlane (n=89) & P value \\
\hline Anterior & $55.65 \pm 34.14$ & $139.5 \pm 62.84$ & 0.002 \\
\hline Posterior & $44.72 \pm 25.33$ & $48.87 \pm 51.58$ & 0.256 \\
\hline Right & $42.78 \pm 28.65$ & $73.29 \pm 50.64$ & 0.023 \\
\hline Left & $55.75 \pm 41.99$ & $64.0 \pm 38.1$ & 0.377 \\
\hline Inferior & $80.33 \pm 45.85$ & $46.0 \pm 12.72$ & 0.042 \\
\hline & & & 0 \\
Superior & 0 & $67.4 \pm 51.29$ & 0.111 \\
\hline
\end{tabular}

Table 4. Average times need for 2D ultrasound vs. live xPlane according to fetal spine position.

Data is presented as number (percentage) 


\section{Table 5 (on next page)}

Feasibilty of 2D ultrasound vs. live xPlane according to operator level

Data is presented as number (percentage). 
Table 5. Feasibilty of 2D ultrasound vs. live xPlane according to operator level.

\begin{tabular}{|c|c|c|c|}
\hline First level operator & 2D ultrasound & Live xPlane & p value \\
\hline 4 Chamber- & & $40 / 60(66.6)$ & $<0.001$ \\
\hline Aortic Arch & $60 / 60(100)$ & & \\
\hline 4 Chamber- & 60/60 (100) & 41/60 (68.3) & $<0.001$ \\
\hline Ductal Arch & 2D ultrasound & Live xPlane & p value \\
\hline Second level operator & & & \\
\hline 4 Chamber- & 54/54(100) & $52 / 54(96.29)$ & 0.56 \\
\hline Aortic Arch & & & \\
\hline 4 Chamber- & $54 / 54(100)$ & $52 / 54(96.29)$ & \\
\hline Ductal Arch & & & \\
\hline
\end{tabular}

Data is presented as number (percentage) 


\section{Table 6(on next page)}

Average of times with 2D ultrasound vs. live xPlane according to operator level

Data is presented as mean $\pm \mathrm{SD}$. 
Table 6. Average of times with 2D ultrasound vs. live xPlane according to operator level.

\begin{tabular}{|c|c|c|c|}
\hline First level operator & 2D ultrasound & Live xPlane & $p$ value \\
\hline $\begin{array}{c}4 \text { Chamber- } \\
\text { Aortic Arch (sec) }\end{array}$ & $30.25 \pm 29.25$ & $51.62 \pm 36.89$ & 0.002 \\
\hline $\begin{array}{c}4 \text { Chamber- } \\
\text { Ductal Arch (sec) }\end{array}$ & $25.51 \pm 20.62$ & $39.09 \pm 34.23$ & 0.03 \\
\hline Second level operator & 2D ultrasound & Live xPlane & $p$ value \\
\hline $\begin{array}{c}4 \text { Chamber- } \\
\text { Aortic Arch (sec) }\end{array}$ & $28.79 \pm 28.17$ & $35.5 \pm 37.9$ & 0.02 \\
\hline $\begin{array}{c}4 \text { Chamber- } \\
\text { Ductal Arch (sec) }\end{array}$ & $18.38 \pm 13.21$ & $35.5 \pm 33.8$ & 0.001 \\
\hline
\end{tabular}

Data is presented as mean $\pm \mathrm{SD}$ 